\title{
Comportamentos de estilo de vida saudável de estudantes universitários e fatores relacionados
}

Healthy lifestyle behaviors of university students and related factors

Comportamientos de estilo de vida saludable de estudiantes universitarios y factores relacionados

Serap Çetinkaya ${ }^{1}$ io https://orcid.org/0000-0001-7706-4748

Havva Sert ${ }^{1}$ id https://orcid.org/0000-0002-1658-6515

Como citar:

Çetinkaya S, Sert H. Comportamentos de estilo

de vida saudável de estudantes universitários

e fatores relacionados. Acta Paul Enferm. 2021;34:AAPE02942.

DOI

http://dx.doi.org/10.37689/actaape/2021A002942

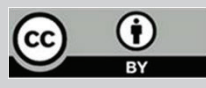

Descritores

Comportamento; Estilo de vida; Universidades;

Estudantes

Keywords

Behavior; Lifestyle; Universities; Students

Descriptores

Conducta; Estilo de vida; Universidades;

Estudiantes

Submetido

19 de Outubro de 2019

Aceito

1 de Julho de 2020

\section{Autor correspondente}

Havva SERT

E-mail: hsert@skarya.edu.tr

\section{Resumo}

Objetivo: Determinar comportamentos saudáveis de estilo de vida de estudantes universitários e fatores relacionados.

Métodos: Projetado para usar o método de amostragem descritivo e estratificado, o estudo incluiu 2.100 estudantes nos departamentos de Ciências da Saúde, Ciências, Ciências Sociais e Ciências da Educação da Sakarya University, Turquia. 0 estudo foi realizado entre maio e dezembro de 2015. Foram coletados dados que incluem características sociodemográficas e Health-Promoting Lifestyle Profile II (HPLP II). 0 teste U de Mann Whitney, 0 teste de Kruskal-Wallis e a correlação de classificação de Spearman foram utilizados para a análise dos dados.

Resultados: 0 escore mediano dos alunos do HLBS II foi de 2,42 e, para as subdimensões, o escore mediano foi de 2,22 para a responsabilidade em saúde (RS), 2,12 para atividade física (AF), 2,22 para nutrição (N), 2,77 para desenvolvimento espiritual (DE), 2,77 para relacionamento interpessoal (RI) e 2,37 para gerenciamento de estresse (GE). Observou-se que sexo, idade, renda, escolaridade, tabagismo, hábitos nutricionais, índice de massa corporal, participação em cursos relacionados à saúde afetam comportamentos de estilo de vida saudáveis.

Conclusão: Observou-se que os alunos apresentaram comportamentos menos responsáveis em relação à atividade física, responsabilidade à saúde e hábitos nutricionais. Para proteger e melhorar a saúde dos alunos, pode ser útil tornar obrigatórios os cursos de promoção da saúde no currículo de todos os departamentos da universidade.

\section{Abstract}

Objective: This study was conducted to determine healthy lifestyle behaviors of university students and related factors.

Methods: Designed to use the descriptive and stratified sampling method, the study included 2100 students studying in the departments of Health Sciences, Science, Social Sciences, and Educational Sciences at Sakarya University, Turkey. The study was conducted between May 2015 and December 2015. Data including socio-demographic characteristics and Health-Promoting Lifestyle Profile II (HPLP II) were collected. The Mann Whitney U test, Kruskal-Wallis test, and Spearman's rank correlation were used for data analysis.

Results: The median score of the students on the HLBS II was 2.42 and for the sub-dimensions, the median score was 2.22 for the health responsibility (HR), 2.12 for physical activity (PA), 2.22 for nutrition (N), 2.77 for spiritual development (SD), 2.77 for interpersonal relationships (IR), and 2.37 for stress management (SM). It was observed that gender, age, income, grade level, smoking, nutritional habits, body mass index, attending courses related to health effect healthy lifestyle behaviors. 
Conclusion: It was observed that students showed the least responsible behaviors with regards to physical activity, health responsibility, and nutritional habits. In order to protect and improve the health of students, it may be useful to make health promotion courses compulsory in the curriculum of all departments in the university.

\section{Resumen}

Objetivo: Determinar comportamientos saludables de estilo de vida de estudiantes universitarios y factores relacionados.

Métodos: El estudio, diseñado para usar el método de muestreo descriptivo y estratificado, incluyó 2.100 estudiantes de los departamentos de Ciencias de la Salud, Ciencias, Ciencias Sociales y Ciencias de la Educación de la Sakarya University, Turquía. El estudio fue realizado entre mayo y diciembre de 2015. Se recolectaron datos que incluyeron características sociodemográficas y Health-Promoting Lifestyle Profile II (HPLP II). Para el análisis de datos, se utilizó la prueba U de Mann-Whitney, la prueba de Kruskal-Wallis y la correlación de clasificación de Spearman.

Resultados: La puntuación mediana de los alumnos en el HLBS II fue 2,42 y, en las subdimensiones, la puntuación mediana fue 2,22 en responsabilidad en salud (RS), 2,12 en actividad física (AF), 2,22 en nutrición (N), 2,77 en desarrollo espiritual (DE), 2,77 en relaciones interpersonales (IR) y 2,37 en manejo del estrés (ME). Se observó que el sexo, la edad, los ingresos, la escolaridad, el tabaquismo, los hábitos nutricionales, el índice de masa corporal y la participación en cursos relacionados con la salud afectan comportamientos saludables de estilo de vida.

Conclusión: Se observó que los alumnos presentan comportamientos menos responsables con relación a la actividad física, la responsabilidad en salud y los hábitos nutricionales. Para proteger y mejorar la salud de los alumnos, puede ser útil hacer obligatorios los cursos de promoción de la salud en el diseño curricular de todos los departamentos de la universidad.

\section{Introdução}

A Organização Mundial da Saúde definiu saúde como um estado de completo bem-estar físico, mental e social e não apenas a ausência de doença ou enfermidade. ${ }^{(1)}$ Com relação ao surgimento de um estado de completo bem-estar, os indivíduos da sociedade precisam assumir a responsabilidade de proteger, desenvolver e gerenciar sua própria saúde. Estilo de vida saudável é definido como controlar todos os comportamentos que podem afetar a saúde do indivíduo e organizar suas atividades diárias, escolhendo os comportamentos adequados à sua própria saúde. ${ }^{(2,3)}$ Afirma-se que um estilo de vida saudável é um componente da promoção da saúde. O estilo de vida saudável deve incluir não apenas a proteção contra doenças, mas também a demonstração de comportamentos que aumentam o nível de bem-estar ao longo da vida. Esses comportamentos são comportamentos auto-realizáveis que aumentam o nível de bem-estar do indivíduo; nutrição equilibrada e adequada, controle do estresse, exercícios adequados e regulares, não fumantes, responsabilidade com a saúde. A fim de prevenir doenças causadas pelo estilo de vida e mortes devido a essas doenças, os indivíduos devem assumir a responsabilidade em termos de comportamentos de estilo de vida saudáveis. ${ }^{(2-4)}$ A vida universitária é um período durante, o qual, novas responsabilidades surgem como resultado de estar longe da família. Mudanças significativas são experimentadas, o que exige que os alunos tenham um papel mais ativo na tomada de decisões relacionadas a si mesmos. ${ }^{(4)}$ Além disso, é também um momento em que o desenvolvimento espiritual, em que os relacionamentos interpessoais e o gerenciamento do estresse começam a se desenvolver. $^{(4-6)}$ A vida universitária é um período muito importante e produtivo em termos de determinação do comportamento dos alunos para proteger e desenvolver sua saúde, alterar comportamentos errados, garantir a continuação de seus bons comportamentos e obter comportamentos de estilo de vida saudáveis. ${ }^{(3)}$ Como a responsabilidade com a saúde, a atividade física, os hábitos nutricionais, o desenvolvimento espiritual, o relacionamento interpessoal e o gerenciamento do estresse, que constituem comportamentos de estilo de vida saudáveis, geralmente começam a se formar durante os anos universitários, os estudos relatados na literatura sobre esses tópicos geralmente são realizados no campo das Ciências da Saúde, e, atualmente, não há estudos envolvendo todos os departamentos da universidade. Este estudo foi realizado para determinar comportamentos saudáveis de estilo de vida de estudantes e fatores relacionados.

\section{Métodos}

O estudo foi aprovado pelo conselho de ética da instituição (Reitor da Faculdade de Medicina da Sakarya University) e realizado de acordo com 
os padróes éticos estabelecidos na Declaração de Helsinque de 1964 e suas alteraçóes posteriores ou padróes éticos comparáveis. Além disso, o consentimento informado foi obtido de todos os participantes individuais do estudo. $\mathrm{O}$ estudo foi realizado utilizando um método de amostragem descritivo e estratificado, com alunos dos departamentos de Ciências da Saúde, Ciências, Ciências Sociais e Ciências da Educação da Sakarya University. A universidade oferece graduação associada e ensino de graduação ao aluno que se ofereceu para participar do estudo. A população do estudo consistiu em 55.732 pessoas estudando na Sakarya University entre maio de 2015 e dezembro de 2015; considerando que a amostra do estudo compreendeu 2.100 estudantes que concordaram voluntariamente em participar do estudo e que não tiveram dificuldades de comunicação. $\mathrm{O}$ número de alunos que compóem a amostra foi calculado multiplicando o número de itens (52) do Health-Promoting Lifestyle Profile II, que apresenta o maior número de itens, por $35(52 \times 35=1820)$. Para que a amostra representasse a sociedade, os estudantes da universidade foram selecionados usando o método de amostragem aleatória estratificada por faculdade. $O$ peso da camada foi calculado dividindo-se o número obtido de 1.820 em 55.732 alunos que estudavam na universidade $(1.820 / 55.732=0,03)$. O tamanho de cada amostra foi calculado multiplicando o número de alunos em cada sub-estrato pelo peso do estrato. Todos os participantes que responderam ao questionário e ao Health-Promoting Lifestyle Profile II foram incluídos. Desenvolvido à luz da literatura pelos pesquisadores para determinar as características sociodemográficas e de estilo de vida dos estudantes, o Health-Promoting Lifestyle Profile II foi usado para coletar os dados. O questionário e a escala foram preenchidos em cerca de 20 minutos por cada participante. Durante a coleta dos dados, foi marcada uma consulta para o dia em que o gerente de cada unidade estava disponível, e os questionários foram distribuídos nos momentos em que os palestrantes e os alunos estavam disponíveis, considerando o currículo do dia. Os estudantes foram informados sobre o estudo, e seu consentimento foi obtido antes do preenchimento do questionário. Em seguida, eles foram convidados a preencher o questionário sozinhos. As medidas antropométricas dos estudantes foram realizadas pelo pesquisador. O questionário geral foi composto por 15 perguntas sobre sexo, idade, nível de renda, classe, tabagismo, nutrição, índice de massa corporal (IMC) e se os alunos estavam recebendo aulas sobre promoção da saúde. A HPLP II, desenvolvida, por Walker et al., é uma escala do tipo Likert de 4 pontos, composta por 52 itens, incluindo responsabilidade em saúde (RS), atividade física (AF), nutrição $(\mathrm{N})$, desenvolvimento espiritual (DE), relacionamento interpessoal (RI) e gerenciamento de estresse (GE) e 6 subdimensóes. Um aumento nas pontuaçóes obtidas na escala indica que os indivíduos aplicam melhor comportamentos de estilo de vida saudáveis. ${ }^{(7-9)} \mathrm{O}$ estudo turco de validade e confiabilidade do HPLP II foi realizado, com dois estudos separados realizados em anos diferentes. Nos estudos de validade e confiabilidade realizados em 2008 e 2009, o coeficiente alfa de Cronbach da escala foi de 0,92 com base em toda a escala. ${ }^{(7)}$ No presente estudo, foi determinado que o coeficiente Alfa de Cronbach na escala foi de 0,88 e que o coeficiente Alfa de Cronbach variou de 0,61 a 0,79 nas subdimensóes da escala. $O$ teste de normalidade foi realizado para determinar os testes a serem utilizados na avaliação dos dados coletados. Como o resultado do teste de normalidade determinou que os escores HPLP II não atingiam a distribuição normal, os testes não paramétricos, Mann Whitney U e Kruskal Wallis H, foram utilizados para analisar os dados. Além disso, também usamos a análise de correlação de Pearson para investigar a associação entre HPLP II e suas subdimensões.

\section{Resultados}

A idade média dos alunos participantes do estudo foi de 20,69 $\pm 2,48$-. Um total de 50,3\% dos estudantes era do sexo masculino $(n=1.044), 72 \%$ deles possuíam renda média (1.512), 49,2\% estudavam em departamentos do instituto de ciências sociais (1.033) e 35\% estavam estudando na segunda série (734). Um total de 53\% dos estudantes nunca havia fumado (1.108), 55\% deles comiam comida casei- 
ra (1.155), 19,1\% geralmente consumiam fast food (401), 68,62\% eram indivíduos com peso normal (1.441) e $79 \%$ tinham ou estavam participando de um curso relacionado à promoção da saúde (430). A mediana dos escores do HPLP II dos estudantes foi de 2,42 e, para as subdimensóes, o escore mediano foi de 2,22 para RS, 2,12 para AF, 2,22 para $\mathrm{N}, 2,72$ para $\mathrm{N}, 2,77$ para $\mathrm{DE}, 2,77$ para $\mathrm{RI}$ e 2,37 para GE. Observou-se que os alunos obtiveram o maior escore mediano para as subdimensóes DE e RI e o menor escore mediano para a subdimensão AF. Quando comportamentos saudáveis de estilo de vida foram avaliados como um todo, determinou-se que a variável sexo não afetou a HPLP II dos alunos ( $p>0,05)$ (Tabela 1). Embora tenha sido observado que as fêmeas apresentaram comportamentos mais desejáveis do que os homens nas áreas de RS e AF das subdimensóes HPLP II e HPLP II, os machos foram considerados melhores na subdimensão RI $(p<0,05)$ (Tabela 1). De acordo com a correlação de Spearman, houve um nível muito alto de relaçóes positivas $(r=0,077, p=0,000)$, significativas entre a idade dos alunos e a subdimensão de RI. O nível de responsabilidade em saúde aumentou à medida que a idade aumentou (não apresentado na tabela). Foi encontrada uma relação moderadamente significativa negativa entre a subdimensão RI e a idade ( $r=-0,050$, $\mathrm{p}=0,022$ ) (não apresentado na tabela). O status da renda foi determinado para afetar estatisticamente significativamente as subdimensóes gerais do HPLP II, N e RI. Observou-se também que os hábitos nutricionais $(\mathrm{p}=0,022)$ e os comportamentos gerais de estilo de vida saudável $(p=0,039)$ daqueles com baixa renda eram mais pobres em comparação com aqueles com alta renda. Na subdimensão de RI do HPLP II, verificou-se que o RI foi estatisticamente significativamente melhor para aqueles com alto status de renda em comparação com aqueles com baixo status de renda $(0,011)$ e status de média renda $(0,05)$. Foi encontrada diferença significativa entre o nível de escolaridade e a subdimensão da RS ( $p<0,05)$. Em uma análise adicional realizada para determinar o grupo que causou a diferença significativa, determinou-se que os comportamentos relacionados à $\mathrm{RS}$ dos alunos da terceira série eram estatisticamente significativamente melhores que os da primeira série $(p=0,001)(p<0,05)$. Em relação ao tabagismo e comportamentos saudáveis de estilo de vida, a variável tabagismo afetou significativamente os comportamentos gerais de estilo de vida saudável e todas as subdimensões além da AF e N ( $\mathrm{p}<0,05)$ (Tabela 1). Também foi determinado que os fumantes do dia-a-dia apresentaram comportamentos estatisticamente mais negativos nas subdimensóes DE $(p=0,015)$, RI $(\mathrm{p}=0,001)$, GE $(\mathrm{p}=0,002)$ e HPLP II geral $(\mathrm{p}=0,007)$, comparado a não fumantes. Foi encontrada diferença estatisticamente significante entre os hábitos nutricionais dos alunos, o escore total das HPLP II e as sub-dimensóes RI, AF, N e GE ( $p<0,05)$. Observou-se também que os estudantes que ingeriram alimentos caseiros aplicaram melhor os comportamentos do estilo de vida saudável em comparação aos estudantes que ingeriram fast-food $(\mathrm{p}=0,000)$ e alimentos em dormitórios/ universidades $(\mathrm{p}=0,039)$. Também foi determinado que os estudantes que ingeriram alimentos caseiros apresentaram melhores escores de RS ( $\mathrm{p}=0,001)$, DE $(0,023)$ e RI $(p=0,028)$ em comparação aos que consomem fast food. Além disso, os comportamentos $\mathrm{Ne}$ GE dos estudantes que preferiram alimentos caseiros foram melhores em comparação aos que consumiam fast food ( $\mathrm{p}=0,000, \mathrm{p}=0,000$, respectivamente) e alimentos para dormitórios/universidades $(p=0,000$, $\mathrm{p}=0,000$, respectivamente). Os comportamentos de AF daqueles com nutrição no estilo fast food foram significativamente menores quando comparados aos que consumiam alimentos caseiros $(\mathrm{p}=0,001)$ e dormitórios/universidades $(p=0,001)$. Foi encontrada diferença estatisticamente significante entre o IMC e o desenvolvimento espiritual e as subdimensóes da AF $(p<0,05)$ (Tabela 1). De acordo com os resultados da análise realizada para determinar o grupo que causou significância, determinou-se que o nível de atividade física de alunos fracos era significativamente menor $(p=0,005)$ em relação aos indivíduos com peso normal. Estudantes com sobrepeso/obesidade apresentaram pior DE em comparação com indivíduos com peso normal $(\mathrm{p}=0,036)$. Os resultados também mostram que aqueles que frequentam cursos relacionados à saúde praticaram comportamentos mais positivos em todas as subdimensóes e HLBS geral em comparação com aqueles que não estavam frequentando cursos relacionados à saúde $(\mathrm{p}<0,05)$ (Tabela 1). 
Tabela 1. Atitudes de estilo de vida saudável dos alunos sobre características descritivas $(n=2.100)(n=2.100)$

\begin{tabular}{|c|c|c|c|c|c|c|c|}
\hline \multirow[b]{2}{*}{ Características } & \multirow{2}{*}{$\begin{array}{c}\text { HPLP II } \\
\text { Mediana (IIQ) }\end{array}$} & \multicolumn{6}{|c|}{ SUB-ESCALA HPLP I| } \\
\hline & & $\begin{array}{c}\text { RS } \\
\text { Mediana (IIQ) }\end{array}$ & $\begin{array}{c}\text { AF } \\
\text { Mediana (IIQ) }\end{array}$ & $\begin{array}{c}\mathrm{N} \\
\text { Mediana (IIQ) }\end{array}$ & $\begin{array}{c}\text { DE } \\
\text { Mediana (IIQ) }\end{array}$ & $\begin{array}{c}\mathrm{Rl} \\
\text { Mediana (IIQ) }\end{array}$ & $\begin{array}{c}\text { GE } \\
\text { Mediana (IIQ) }\end{array}$ \\
\hline \multicolumn{8}{|l|}{ Sexo } \\
\hline Feminino (1044) & $2,42(0,46)$ & $2,22(0,67)$ & $2,25(0,75)$ & $2,22(0,56)$ & $2,77(0,56)$ & $2,66(0,78)$ & $2,37(0,63)$ \\
\hline \multirow[t]{2}{*}{ Masculino (1056) } & $2,42(0,48)$ & $2,22(0,56)$ & $2,00(0,88)$ & $2,22(0,56)$ & $2,77(0,56)$ & $2,77(0,67)$ & $2,37(0,75)$ \\
\hline & $\begin{array}{c}z=-0,122 \\
p=0,90\end{array}$ & $\begin{array}{l}z=-2,641 \\
p=0,008^{\star *}\end{array}$ & $\begin{array}{c}\mathrm{z}=-8,437 \\
\mathrm{p}=0,000^{\star \star \star}\end{array}$ & $\begin{array}{l}z=-0,049 \\
p=0,961\end{array}$ & $\begin{array}{l}z=-1,764 \\
p=0,078\end{array}$ & $\begin{array}{l}z=-5,217 \\
p=0,000^{\star \star \star}\end{array}$ & $\begin{array}{l}z=-0,262 \\
p=0,794\end{array}$ \\
\hline \multicolumn{8}{|l|}{ Nível de renda } \\
\hline Bom (463) & $2,46(0,48)$ & $2,22(0,67)$ & $2,25(0,88)$ & $2,33(0,56)$ & $2,88(0,67)$ & $2,77(0,78)$ & $2,37(0,63)$ \\
\hline Médio (1512) & $2,42(0,46)$ & $2,22(0,67)$ & $2,12(0,88)$ & $2,22(0,56)$ & $2,77(0,56)$ & $2,77(0,67)$ & $2,37(0,63)$ \\
\hline \multirow[t]{2}{*}{ Ruim (125) } & $2,38(0,51)$ & $2,11(0,67)$ & $2,25(0,88)$ & $2,11(0,88)$ & $2,77(0,67)$ & $2,77(0,72)$ & $2,25(0,63)$ \\
\hline & $\begin{array}{l}x^{2}=7,755 \\
p=0,021^{*}\end{array}$ & $\begin{array}{l}x^{2}=10,262 \\
p=0,006^{\star \star}\end{array}$ & $\begin{array}{l}x^{2}=5,127 \\
p=0,077\end{array}$ & $\begin{array}{l}x^{2}=7,592 \\
p=0,022^{*}\end{array}$ & $\begin{array}{l}x^{2}=5,315 \\
p=0,070\end{array}$ & $\begin{array}{l}x^{2}=5,382 \\
p=0,068\end{array}$ & $\begin{array}{l}x^{2}=3,796 \\
p=0,150\end{array}$ \\
\hline \multicolumn{8}{|l|}{ Classe } \\
\hline Primeira (710) & $2,42(0,51)$ & $2,11(0,67)$ & $2,25(0,88)$ & $2,22(0,56)$ & $2,88(0,56)$ & $2,78(0,67)$ & $2,37(0,75)$ \\
\hline Segunda (734) & $2,42(0,44)$ & $2,22(0,67)$ & $2,12(0,75)$ & $2,22(0,56)$ & $2,78(0,56)$ & $2,78(0,67)$ & $2,37(0,63)$ \\
\hline Terceira (317) & $2,42(0,46)$ & $2,22(0,78)$ & $2,25(0,75)$ & $2,33(0,56)$ & $2,78(0,67)$ & $2,67(0,67)$ & $2,25(0,63)$ \\
\hline \multirow[t]{2}{*}{ Quarta (339) } & $2,42(0,44)$ & $2,22(0,67)$ & $2,12(0,81)$ & $2,22(0,56)$ & $2,78(0,44)$ & $2,67(0,67)$ & $2,37(0,63)$ \\
\hline & $\begin{array}{c}x^{2}=0,109 \\
p=0,99\end{array}$ & $\begin{array}{l}x^{2}=14,354 \\
p=0,002^{\star \star}\end{array}$ & $\begin{array}{c}x^{2}=3,893 \\
p=0,27\end{array}$ & $\begin{array}{l}x^{2}=1,981 \\
p=0,580\end{array}$ & $\begin{array}{l}x^{2}=4,626 \\
p=0,201\end{array}$ & $\begin{array}{l}x^{2}=7,349 \\
p=0,062\end{array}$ & $\begin{array}{l}x^{2}=0,279 \\
p=0,970\end{array}$ \\
\hline \multicolumn{8}{|l|}{ Tabagismo } \\
\hline Eu nunca fumei (1108) & $2,44(0,46)$ & $2,22(0,67)$ & $2,12(0,88)$ & $2,22(0,56)$ & $2,89(0,56)$ & $2,78(0,67)$ & $2,37(0,63)$ \\
\hline Eu fumo todos os dias (647) & $2,40(0,78)$ & $2,22(0,78)$ & $2,25(0,78)$ & $2,22(0,67)$ & $2,78(0,67)$ & $2,67(0,78)$ & $2,25(0,63)$ \\
\hline Eu uso ocasionalmente (273) & $2,40(0,78)$ & $2,22(0,78)$ & $2,12(0,88)$ & $2,22(0,56)$ & $2,78(0,56)$ & $2,78(0,67)$ & $2,37(0,63)$ \\
\hline \multirow[t]{2}{*}{ Parei de fumar (72) } & $2,46(0,56)$ & $2,17(0,56)$ & $2,31(0,88)$ & $2,22(0,75)$ & $2,78(0,44)$ & $2,78(0,75)$ & $2,44(0,72)$ \\
\hline & $\begin{array}{l}x^{2}=11,010 \\
p=0,012^{\star}\end{array}$ & $\begin{array}{l}x^{2}=9,003 \\
p=0,059^{\star}\end{array}$ & $\begin{array}{l}x^{2}=5,850 \\
p=0,119\end{array}$ & $\begin{array}{l}x^{2}=5,165 \\
p=0,160\end{array}$ & $\begin{array}{l}x^{2}=9,946 \\
p=0,019^{*}\end{array}$ & $\begin{array}{l}x^{2}=14,542 \\
p=0,002^{* *}\end{array}$ & $\begin{array}{l}x^{2}=14,089 \\
p=0,003^{\star *}\end{array}$ \\
\hline \multicolumn{8}{|l|}{ Nutrição } \\
\hline Geralmente fast food (401) & $2,35(0,48)$ & $2,11(0,67)$ & $2,12(0,75)$ & $2,11(0,56)$ & $2,78(0,67)$ & $2,78(0,78)$ & $2,25(0,63)$ \\
\hline Comida em casa (1155) & $2,46(0,46)$ & $2,22(0,67)$ & $2,25(0,75)$ & $2,33(0,56)$ & $2,89(0,56)$ & $2,78(0,67)$ & $2,37(0,63)$ \\
\hline \multirow{2}{*}{$\begin{array}{l}\text { Refeições preparadas na } \\
\text { universidade/dormitório (544) }\end{array}$} & $2,46(0,46)$ & $2,22(0,67)$ & $2,25(0,84)$ & $2,22(0,67)$ & $2,78(0,56)$ & $2,78(0,78)$ & $2,37(0,63)$ \\
\hline & $\begin{array}{l}x^{2}=33,782 \\
p=0,000^{\star \star \star}\end{array}$ & $\begin{array}{l}x^{2}=12,810 \\
p=0,002^{\star \star}\end{array}$ & $\begin{array}{l}x^{2}=17,624 \\
p=0,000^{\star \star \star}\end{array}$ & $\begin{array}{l}x^{2}=56,795 \\
p=0,000^{\star \star \star}\end{array}$ & $\begin{array}{l}x^{2}=7,567 \\
p=0,023^{*}\end{array}$ & $\begin{array}{l}x^{2}=6,895 \\
p=0,032^{*}\end{array}$ & $\begin{array}{l}x^{2}=16,530 \\
p=0,001^{\star \star}\end{array}$ \\
\hline \multicolumn{8}{|l|}{ IMC } \\
\hline Abaixo do peso (257) & $2,42(0,47)$ & $2,22(0,67)$ & $2,12(0,88)$ & $2,22(0,67)$ & $2,78(0,56)$ & $2,78(0,67)$ & $2,37(0,75)$ \\
\hline Normal (1441) & $2,42(0,46)$ & $2,22(0,67)$ & $2,25(0,75)$ & $2,22(0,56)$ & $2,78(0,56)$ & $2,78(0,67)$ & $2,37(0,75)$ \\
\hline \multirow[t]{2}{*}{ Sobrepeso/obesidade (402) } & $2,40(0,44)$ & $2,22(0,67)$ & $2,22(0,56)$ & $2,22(0,56)$ & $2,78(0,56)$ & $2,67(0,78)$ & $2,31(0,63)$ \\
\hline & $\begin{array}{l}x^{2}=4,614 \\
p=0,202\end{array}$ & $\begin{array}{l}x^{2}=5,325 \\
p=0,150\end{array}$ & $\begin{array}{c}x^{2}=10,006 \\
p=0,007\end{array}$ & $\begin{array}{c}x^{2}=7,842 \\
p=0,05\end{array}$ & $\begin{array}{l}x^{2}=6,406 \\
p=0,041\end{array}$ & $\begin{array}{l}x^{2}=4,266 \\
p=0,234\end{array}$ & $\begin{array}{l}x^{2}=1,008 \\
p=0,799\end{array}$ \\
\hline \multicolumn{8}{|l|}{$\begin{array}{l}\text { Situação de tirar lições sobre } \\
\text { promoção da saúde }\end{array}$} \\
\hline $\operatorname{Sim}(430)$ & $2,51(0,52)$ & $2,33(0,67)$ & $2,37(0,75)$ & $2,33(0,67)$ & $2,88(0,67)$ & $2,88(0,78)$ & $2,50(0,63)$ \\
\hline \multirow[t]{2}{*}{ Não (1670) } & $2,40(0,46)$ & $2,22(0,78)$ & $2,12(0,88)$ & $2,22(0,56)$ & $2,77(0,56)$ & $2,66(0,78)$ & $2,37(0,63)$ \\
\hline & $\begin{array}{l}z=-6,32 \\
p=0,000\end{array}$ & $\begin{array}{l}z=-5,73 \\
p=0,000\end{array}$ & $\begin{array}{l}\mathrm{z}=-5,49 \\
\mathrm{p}=0,000\end{array}$ & $\begin{array}{l}\mathrm{z}=-3,55 \\
\mathrm{p}=0,000\end{array}$ & $\begin{array}{l}z=-4,065 \\
p=0,000\end{array}$ & $\begin{array}{l}z=-4,21 \\
p=0,000\end{array}$ & $\begin{array}{l}z=-4,58 \\
p=0,000\end{array}$ \\
\hline
\end{tabular}

IIQ - Intervalo interquartil, z: Mann-Whitney U, x2: teste de Kruskal Wallis $\mathrm{H} ;{ }^{\star} \mathrm{p}<0,05$.

\section{Discussão}

No escopo deste estudo, a pontuação média da HPLP II dos estudantes foi de 2,42. Resultados semelhantes aos obtidos no presente estudo também foram encontrados nos estudos de Beşer et al. 2007,(7) Malakouti et al. 2017,(10) Yüksel Kaçan e Örsal 2019, ${ }^{(11)}$ e Al Khawaldeh 2014. ${ }^{(12)}$ Ao contrário dos resultados do presente estudo, no entanto diferentes resultados foram obtidos nos estudos de Montazeri et al. 2017 $7^{(13)}$ e Thacker et al. 2016. ${ }^{(14)}$

De acordo com os resultados obtidos no presente estudo, os estudantes apresentaram bons níveis de DE e RI e foram inadequados na realização da AF. Resultados semelhantes foram encontrados em vários outros estudos. ${ }^{(4,5,15-19)}$ Em nosso estudo, a razão pela qual os estudantes universitários tiveram um bom desenvolvimento espiritual e relacionamentos interpessoais pode ser o fato de que as 
interaçóes sociais dos estudantes aumentam durante a vida universitária e, portanto, as relaçóes interpessoais ganham mais importância. ${ }^{(20)}$ A razão para um baixo nível de atividade física pode ser o fato de os estudantes universitários considerarem que a atividade física deve ser normalmente aplicada para perda de peso ou devido a doença crônica, e não para proteger e melhorar sua saúde. ${ }^{(21,22)}$

No presente estudo, observou-se que os níveis de $\mathrm{RS}$ e AF das fêmeas eram melhores que os do sexo masculino. Os níveis de RI dos machos foram melhores que as fêmeas. Diferentemente do nosso estudo, foi determinado em um estudo de Karadamar et al. 2014 ${ }^{(23)}$, que os homens atribuíram maior importância à $\mathrm{AF}$ em comparação às mulheres, e em um estudo de Sen et al. 2017, ${ }^{(5)}$ determinou-se que os homens atribuíam maior importância à $A F$ e à RI em comparação às mulheres. Em diferentes estudos, não foi observada diferença significativa na RI de acordo com a variável sexo. ${ }^{(17,23)}$ Quando estudos que apoiam os resultados do presente estudo também são levados em consideração, acreditamos que as mulheres apresentavam uma alta RS devido ao papel das mulheres na cultura turca e ao fato de exibirem atitudes mais protetoras e atenciosas com relação à sua própria saúde e à saúde. ambiente devido a esse papel. ${ }^{(11,19)}$

Nossos resultados mostram que o RI dos alunos aumentou com a idade. Em outros estudos, no entanto, observou-se que a idade não afetou a RS. (7,24,25) $^{-1}$ Diferentemente desses outros estudos, o presente estudo foi realizado não apenas nos departamentos relacionados à saúde, mas também em todos os outros departamentos. A educação sobre proteção e desenvolvimento da saúde é recebida desde a primeira série nos departamentos relacionados à saúde. Em nossa universidade, os alunos podem escolher cursos universitários eletivos comuns na terceira e quarta série em departamentos não relacionados à saúde. Os alunos podem escolher cursos relacionados à saúde ou participar ainda mais de atividades relacionadas à saúde por meio deste aplicativo, o que lhes permite participar de cursos fora de seus próprios campos. Todos esses fatores podem explicar por que a responsabilidade com a saúde aumenta com a idade. Observou-se que estudantes com baixa renda pres- tam menos atenção aos comportamentos de estilo de vida saudáveis e $\mathrm{N}$ comparados àqueles com alta renda. Nesse sentido, os achados de nosso estudo são consistentes com a literatura. ${ }^{(7,16,19)}$

Os resultados do presente estudo mostram que os alunos da terceira série apresentaram maior RS quando comparados aos da primeira série. Em um estudo realizado por Yetgin e Agopya 2017, , $^{(4)}$ foram obtidos resultados semelhantes aos de nosso estudo. No entanto, diferentemente do nosso estudo, no estudo de Karaahmetoğlu et al. 2014, ${ }^{(17)}$ nenhuma mudança foi observada em termos de responsabilidade em saúde entre as séries. Acreditamos que a razão pela qual os alunos da primeira série tenham menos RI do que os da terceira série em nosso estudo é porque os alunos da primeira série passam por um período desafiador de exames antes de entrar na vida universitária. Além disso, são inativos nesse processo, têm mais estresse e não prestam a atenção necessária à sua saúde. ${ }^{26,27)}$

Os estudantes que ingeriram alimentos caseiros aplicaram melhor o HLBS em comparação aos que consomem fast-food e ingerem alimentos em dormitórios/universidades. Além disso, os estudantes que geralmente consomem fast food realizam menos atividade física em comparação com aqueles que comem alimentos caseiros e dormitórios/universidades. Também foi observado que aqueles que ingeriam alimentos caseiros administravam melhor o estresse do que aqueles que ingeriam alimentos em dormitórios/universidades. Não foram encontrados estudos discutindo comportamentos saudáveis de estilo de vida e estilo nutricional. No entanto, identificamos que os alimentos caseiros eram melhores do que todos os outros estilos nutricionais do estudo, porque os alimentos caseiros são mais naturais. Além disso, os estudantes que comem dessa maneira preferem alimentos mais saudáveis e naturais que contêm menos óleo do que alimentos feitos fora de casa. Os estudantes que não preferem alimentos caseiros podem ter mais estresse, pois não sabem o conteúdo dos alimentos que ingerem ou como foram preparados. Além disso, os estudantes que consomem alimentos caseiros podem ter sofrido menos estresse devido ao calor e à confiança no ambiente doméstico e familiar. 
Nosso estudo mostrou que os estudantes que fumavam todos os dias tinham HPLP II mais negativo e pior DE, RI e GE em comparação aos não fumantes. Em um estudo de Tsai et al. 2016, ${ }^{(24)}$ que inclui resultados semelhantes ao presente estudo, determinou-se que os não fumantes se adaptaram mais ao HLBS e apresentaram $\mathrm{N}$ melhor em comparação aos fumantes. Da mesma forma, nos estudos de Bostan e Beşer 2016, ${ }^{(2)}$ observou-se que os alunos tiveram melhor $\mathrm{N}$. O fato é que os indivíduos que evitam maus hábitos que afetam negativamente a saúde, como o tabagismo, também têm consciência de outros comportamentos que protegerão e melhorar sua saúde. ${ }^{(22,25)}$ Observamos que os estudantes que frequentavam cursos relacionados à saúde aplicaram melhor o HPLP II, tiveram mais RS e AF e melhor N, maior nível de DE, boa RI e administraram melhor o estresse em comparação com aqueles que não frequentavam cursos relacionados à saúde. No estudo de Yetgin e Agopyan 2017, (4) foi afirmado que os níveis de AF dos estudantes que frequentam cursos relacionados à saúde eram melhores. A razão para esse resultado pode ser o fato de os cursos serem oferecidos para proteger e melhorar a saúde pública, levando a mudanças comportamentais positivas nos alunos.

No presente estudo, observou-se que o IMC não afetou o HPLP II geral. No entanto, quando as subdimensóes foram avaliadas, os alunos com sobrepeso/obesidade apresentaram melhores níveis de AF e menores níveis de DE. Semelhante a este estudo, muitos outros estudos mostram que o IMC não afetou o HPLP II geral. Em um estudo realizado por Tedik e Hacıalioğlu 2017(25), diferentemente dos resultados do presente estudo, observou-se que os estudantes realizavam menos $\mathrm{AF}$ à medida que o IMC aumentava. $\mathrm{O}$ motivo dessa diferença pode ser o fato de o grupo da amostra e o tamanho da amostra em nosso estudo serem diferentes e que os alunos com sobrepeso/obesidade aumentaram suas atividades físicas para perder peso. ${ }^{(28,29)}$

Os participantes do presente estudo foram compostos apenas por estudantes da Sakarya University, portanto, consideramos que as conclusôes do nosso estudo não podem ser generalizadas para todas as universidades.

\section{Conclusão}

Concluindo, observou-se que os estudantes apresentaram comportamentos menos responsáveis em relação à atividade física, responsabilidade à saúde e hábitos nutricionais. É determinado que sexo, nível de renda, nível de escolaridade, tabagismo, nutrição, IMC, situação de tomar lições sobre promoção da saúde afetam comportamentos saudáveis do estilo de vida. Os estudantes de baixa renda, os recém-formados, os que fumam, os que não comem alimentos caseiros, os alunos com IMC fora da faixa normal e os que não frequentavam cursos relacionados à saúde constituíram os grupos que puderam beneficiar de intervençôes de estilo de vida saudável. Para proteger e melhorar a saúde dos estudantes, pode ser útil tornar obrigatórios os cursos de promoção da saúde no currículo de todos os departamentos da universidade. Além disso, recomenda-se aumentar o número de restaurantes que servem comidas caseiras dentro e fora da universidade e garantir que eles forneçam serviços econômicos aos estudantes. Finalmente, abrir clínicas de abandono do tabagismo nas instituiçôes de saúde da universidade também pode ser benéfico.

\section{Colaborações}

Çetinkaya S e Sert H declaram que contribuíram para o desenho do estudo, análise e interpretação dos dados, redação do artigo e aprovação da versão final a ser publicada.

\section{Referências}

1. World Health Organization (WHO). WHO remains firmly committed to the principles set out in the preamble to the Constitution. Geneve: WHO; 2018. [cited 2020 Jun 30]. Available from https://www.who.int/ about/who-we-are/constitution

2. Bostan N, Beşer A. Factors affecting the healthy lifestyle behaviors of nurses. J Educ Res Nurs. 2017;14(1):38-44.

3. Duran Ü, Öğüt S, Asgarpour H, Kunter D. Evaluation of the health personnel's healthy lifestyle behaviors. Adnan Menderes Univ Faculty Health Sci J. 2017;2(3):138-47.

4. Yetgin MK, Agopyan A. Healthy lifestyle behaviours of sports sciences faculty students. Spormetre. 2017;15(3):177-84. 
5. Şen MA, Ceylan A, Kurt ME, Palancı Y, Adın C. Healthy lifestyle behaviours of vocational school of health services students and influential factors. Dicle Med J. 2017;44(1):1-12.

6. Mašina T, Madžar T, Musil V, Milošević M. Differences in healthpromotıng lıfestyle profıle among croatıan medıcal students accordıng to gender and year of study. Acta Clin Croat. 2017;56(1):84-91.

7. Bahar Z, Beşer A, Gördes N, Ersin F, KIssal A. Healthy life style behavior scale $\|: A$ reliability and validity study. Cumhuriyet Univ J Nurs. 2008;12(1):1-13.

8. Pinar R, Celik R, Bahcecik N. Reliability and construct validity of the Health-Promoting Lifestyle Profile II in an adult Turkish population. Nurs Res. 2009 ;58(3):184-93.

9. Karakoç Kumsar A, Çınar Pakyüz S. Determining the healthy lifestyle behaviors and related factors on women with metabolic syndrome. Koç Univ J Nurs Educ Res. 2015;12(1):19-24.

10. Malakouti J, Sehhati F, Mirghafourvand M, Nahangi R. Relationship between health promoting lifestyle and perceived stress in pregnant women with preeclampsia. J Caring Sci. 2015;4(2):155-63.

11. Yüksel Kaçan C, Örsal Ö. Evaluation of healthy life style behaviors level of nursing students. J Duzce Univ Health Sci Inst. 2019;9(1):19-24.

12. Al-Khawaldeh OA. Health promoting lifestyles of Jordanian university students. Int J Adv Nurs Stud. 2014;3(1):27-31.

13. Montazeri N, Kianipour N, Nazari B, Ziapour A, Bakhshi S. Health promoting behaviors among university students: a case-sectional study of Kermanshah University of Medical Sciences. Int J Pediatr. 2017;5(6):5091-9.

14. Thacker K, Haas Stavarski D, Brancato V, Flay C, Greenawald D. CE: Original research: An investigation into the health-promoting lifestyle practices of RNs. Am J Nurs. 2016;116(4):24-30.

15. Çalışkan $C$, Arberk K, Üner $S$. Healthy lifestyle behaviours of university students. Turk J Public Health. 2018;16(3):204-13.

16. Solak Karabörk S. Evaluatıon of healthy Iıving behavior and effectıve factors: smokıng ındıvıduals. Kahramanmaraş: Kahramanmaraş Sütçü İmam University Faculty of Medicine; 2016.
17. Karaahmetoğlu UG, Soğuksu S, Softa Kaçan H. Investıgatıng healthy lıfe style behaviours of first and fourth year nursing students and affecting factors. J Erciyes Univ Fac Health Sci. 2014;2(2):26-42.

18. UzD, KitişY. Determınatıon of healthy lıfe style behavıors and self-effıcacy of nurses workıng in a hospital. Gazi J Health Sci. 2017;2(3):27-39.

19. Shaheen AM, Nassar OS, Amre HM, Hamdan-Mansour AM. Factors affecting health-promoting behaviors of university students in Jordan. Health. 2015;7(01):1-8.

20. Karahan F, Sardoğan ME, Özkamalı E, Dicle AN. Investıgatıon of university compliance level students 'university compliance levels for sociocultural actıvitıes. J Çukurova Univ Fac Educ. 2005;2(30):63-72.

21. YIImazA,MehmetU. Making goals physical activity of women and challenges faced in recreactional areas. Hacettepe J Sport Sci. 2016;27(3):101-17.

22. Oğuz S, Çamcı G, Yılmaz RK. State of university students' physical activity and knowing the effect of physical activity on heart health. Gümüshane Univ J Health Sci. 2018;7(1):54-61.

23. Karadamar M, Yiğit R, Sungur MA. Evaluation of healthy lifestyle behaviours in adolescents. J Anatolia Nurs Health Sci. 2014;17(3):131-9.

24. Tsai SL, Lai CL, Chi MC, Chen MY. Cigarette smoking and healthpromoting behaviours among tuberculosis patients in rural areas. $J$ Clin Nurs. 2016;25(17-18):2511-9.

25. Tedik SE, Hacıalioğlu N. Investıgatıon of the relatıonshıp between excess weight and healthy lifestyle behaviors in nursing students. Int Refer J Nurs Res. 2017;10:59-82.

26. YIImaz HK. Investigation on adolescents in relation to eating attitudes, exam anxiety and depression [ dissertation]. St. Petersburg: Health Sciences Institute; 2015.

27. Gümüş $A B$, Yardımcı $H$, Keser $A$. Evaluation of nutritional situations according to anxiety score of students prepared for exam. J Duzce Univ Health Sci Inst. 2018;8(1):22-8.

28. Akyol A, Yeşilbalkan ÖU, Kutlay E, Kankaya H, Menekli T, Fadiloğlu Ç. The effects of exercise on quality of life and physiological parameters in obese nursing students. Educ Res J Nurs. 2018;15(3):161-9.

29. Yılmaz A. Physical activity, sedantery behaviour and quality of life among university students. Int J Soc Res. 2019;10(17):1433-53. 